\title{
Endocrine Abstracts
}

\begin{tabular}{|c|c|c|c|c|c|c|c|c|}
\hline P023 & $<$ Prev & Next> & ^ Section & ᄎ Contents & Cite & Volume 59 & $<$ & $>$ \\
\hline
\end{tabular}

Search for abstract title, authors etc.

\section{Analysis of diagnosis and growth dynamics of adrenal incidentalomas in a large general hospital}

Miriam Giordano Imbroll ${ }^{1,2}$, Maria Farrugia ${ }^{1}$, Simon Mifsud ${ }^{1}$, Josanne Vassallo ${ }^{1,2}$ \& Mark Gruppetta ${ }^{1,2}$

-35 views f Facebook y Twitter $\rightleftharpoons$ Email + More 它 Print

Author affiliations

Introduction: Adrenal incidentalomas are masses discovered incidentally on imaging studies performed for possible pathologies other than suspected adrenal disease.

Aim: To characterise a cohort of adrenal incidentalomas found on CT.

Methods: This was a retrospective analysis, taking into account all the adrenal incidentalomas discovered on CT between July and December 2014 at the main hospital in Malta. The adrenal lesions were then classified according to these radiological features. CT scans done prior to and after the study period were also reviewed to establish any change in size of the lesions.

Results: Adrenal incidentalomas were identified in 296 patients, out of 9100 CT scans reviewed. Mean age was 66.9 years ( \pm 12 .2S.D.). 97 (33\%) adrenal lesions could not be classified. Of the remaining 199 incidentalomas, 156 (78\%) were confirmed adenomas (Hounsfield units $<10$, relative or absolute washout values of $>40 \%$ or $60 \%$ respectively), 28 (14\%) were metastasis, 12 (6\%) myelolipomas, $3(2 \%)$ ganglioneuromas. In the adenoma group, $49.4 \%$ were males whereas in the metastasis group $71.4 \%$ were males. In the adenoma group, 57\% had a left-sided lesion, 34\% a right-sided lesion and $9 \%$ had bilateral lesions. In the metastasis group $61 \%$ had left sided lesions, $21 \%$ right sided and $18 \%$ bilateral lesions. Largest mean diameter was $20.0 \mathrm{~mm}( \pm 7.4$ S.D. $)$ in the adenoma group and $31.1 \mathrm{~mm}( \pm 18.7$ S.D.) in the metastasis group ( $P=0.033$ ). Median follow up in the adenoma group was 46.3 months (ICR 4.9-96.5) whereas in the metastasis group it was 28 months (ICR 0-28.5). Mean change in size was $0.3 \mathrm{~mm}$ (S.D. \pm 2.0 ) in the adenoma as compared to $20.8 \mathrm{~mm}$ (S.D. \pm 19.7$)$ in the metastasis group ( $P=0.0001)$.

Conclusion: This study continues to confirm that adrenal adenomas are the commonest

We place cookies on your device to give you the best experience of this website. If you don't change your cookie settings, we'll assume you're happy with this.
V vlasguvv, UN

19 Nov 2018 - 21 Nov 2018

Society for Endocrinology_

Browse other volumes

Summary

$\underline{\text { Abstracts }}$

Programme

Volume Editors

Abstract Book

EPosters

Article tools

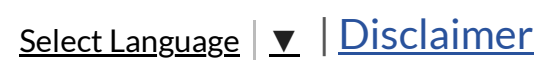

My recent searches

No recent searches.

My recently viewed abstracts

No recent abstracts.

Authors

Imbroll Miriam Giordano

\section{Yes, that's fine}

Change settings 


\section{Endocrine Abstracts}

ISSN 1470-3947 (print) | ISSN 1479-6848 (online)

(c) Bioscientifica 2022 | Privacy_policy | Cookie settings

\section{BiosciAbstracts}

Bioscientifica Abstracts is the gateway to a series of products that provide a permanent, citable record of abstracts for biomedical and life science conferences.

\section{Find out more}

We place cookies on your device to give you the best experience of this website. If you don't change your cookie settings, we'll assume you're happy with this.
Yes, that's fine

Change settings

I want more

information 